\title{
CONSTRUCTAL DESIGN OF A TUBULAR ARRAY SUBJECTED TO FORCED CONVECTION
}

\begin{abstract}
V. A. Pedrotti,
J. A. Souza,

E. D. dos Santos,

and L. A. Isoldi

Universidade Federal do Rio Grande

Laboratório de Simulação Numérica

Escola de Engenharia

Av. Itália $\mathrm{km} 8, \mathrm{~s} / \mathrm{n}$.

CEP 96203-900

Rio Grande, Rio Grande do Sul, Brasil

vagnerpedrotti@,hotmail.com

Received: March 04, 2015

Revised: April 06, 2015

Accepted: May 07, 2015

ABSTRACT

In this work a tubular array (four tubes) subjected to a transverse forced flow is analyzed in terms of thermal performance. Taking into account that there are two main assembles usually used in heat exchanger equipment (aligned and staggered), and that there exist an uncountable number of possible assembles for an array of tubes, present work proposes to use the Constructal Theory to build an optimized assemble. The distance between tubes $(p)$, and the region where tubes can be positioned are the geometric constraint of the problem. Four values for $p$ were considered: $p=1.25 D$ (tube diameter), $p=1.5 D, p=2 D, p$ is free (no restriction). Fluid flow is considered bi-dimensional, incompressible and laminar with $R e_{\mathrm{D}}=10$ and $P r=0.71$. Mass, momentum and energy equations were solved by the Finite Volume Method (FVM) using FLUENT software. Geometry creation and mesh generation were performed with GMSH software while VISIT software was used for the post processing. Results have shown that imposing no restriction to tube positioning do not necessarily lead to best system thermal performance. In this particular study, setting $p=2 D$ has resulted in best thermal performance.

Keywords: tubular assemble, optimization, constructal design
\end{abstract}

\section{NOMENCLATURE}

A area, m

$c_{p} \quad$ specific heat at constant pressure, $\mathrm{J} /(\mathrm{kg} \mathrm{K})$

$h$ convective heat transfer coefficient, $\mathrm{W} /\left(\mathrm{m}^{2} \mathrm{~K}\right)$

$k$ thermal conductivity, $\mathrm{W} /(\mathrm{m} \mathrm{K})$

$p \quad$ distance between tubes, $\mathrm{m}$

$\mathrm{Pr} \quad$ Prandtl number

q heat flux, W

q"' $\quad$ volumetric heat flux, $\mathrm{W} / \mathrm{m}^{3}$

$\operatorname{Re}_{D} \quad$ Reynolds number

$S_{x} \quad$ distance between tubes in $x$ direction, $\mathrm{m}$

$S_{y} \quad$ distance between tubes in $y$ direction, $\mathrm{m}$

$T$ temperature, $\mathrm{K}$

$U$ inlet flow velocity, $\mathrm{m} / \mathrm{s}$

$v \quad$ velocity vector, $\mathrm{m} / \mathrm{s}$

\section{Greek symbols}

$\rho \quad$ density, $\mathrm{kg} / \mathrm{m}^{3}$

$\mu \quad$ absolute viscosity, $\mathrm{Pa} \mathrm{s}$

$\varphi \quad$ control function
Forced flow over an array of heated (or cooled) tubes is an important research area with main applications in heat exchangers design and specification. Such equipment are largely used in air conditioning, airplanes, cars and many industrial processes. Even though a large amount of works can be found in this topic, there are many applications on which the physical phenomena is not completely understood (or modeled) and many other application on which process optimization is needed.

There are many numerical and experimental works about external forced flow over a single tube. Catalano, P. et al. (2003) has studied the application of the wall model boundary condition and concluded that this formulation can be used in simulations with Reynolds number up to $1 \times 10^{6}$. Working with vortex streets, Rajani, B. N. et al. (2009) have investigated laminar and turbulent flows and observed that vortex shedding appearance are less regular and with short wave length when $R e>250$. Evaluating the proximity of the tube with the wall and the occurance of the vortex shedding in this region Sarkar, S. and Sarkar, S. (2010) observed that the stagnation point tends to move toward to the wall creating a force which deviates from the boundary layer.

For the two cylinder case, Sumner, D. (2010) presented a good review of studies that consider a large range of Reynolds numbers and various assembles with different distances between tubes.

In a study of a three tube assemble, Bao, Y. et al. (2010) investigated drag and void formation for Reynolds number equal to 100 . They studied many

\section{INTRODUCTION}


assembles varying tube distance and angle between tubes. This study include comparison between numerical and experimental data.

Works with a greater number of tubes can also be found in literature. Daróczy, L. et al. (2014) investigated an assemble with 7 tubes. The objective was to maximize the heat exchange between the flow stream and tubes with minimal possible pressure drop. Using an Genetic Algorithm (GA), they ran over 140,000 simulations considering tube positioning with and without constraint. Results showed that the inclusion of positioning constraint to the solution algorithm leads to difficult in the GA. Moreover, no advance (better performance) was observed in including any kind of constraint.

Stanescu, G. et al. (1996), presented numerical and experimental results for assembles with 12, 14, 16 and 23 tubes subjected to forced convection. They searched for the optimal distance among tubes for the maximization of the thermal conductivity. Tube occupation area was kept constant and runs were performed for Reynolds number varying from 40 to 2000. Four degrees of freedom (distance between tubes, $S$, over tube diameter, $D$ ) were analyzed: $S / D=$ $0.25,0.5,0.75$ and 1 . Results showed that optimized $S / D$ values decreases with the increase of Reynolds number.

Constructal Design has already been used in thermal optimization applications of tube arrays. Bello-Ochende, T. and Bejan, A. (2005) studied an array of aligned tubes subjected to natural convection. They observed that heat transfer between flow and tubes increases with increasing the complexity of the system. In a following work BelloOchende, T. et al. (2011) studied an array of rotation cylinders of different sizes under forced convection. They concluded that the rotation is beneficial and increases the heat exchange when the tubes are aligned.

Investigations utilizing two tubes aiming to optimize heat transfers and reduce drag have been performed by Rocha, L. A. O. et al. (2013). Results indicated that best performance is obtained when tubes are aligned with an angle varying between $45^{\circ}$ and $60^{\circ}$ to the main fluid flow stream.

Presented review has mentioned just a few number of works related to the study of arrays of tubes subjected to forced convection. As stated before, this is an important area with many engineering applications. Many other works can also be found in literature, however none of them have proposed an deterministic algorithm (procedure) to calculate the tube position that maximizes or minimizes an desired variable (heat transfer, pressure drop, etc.). Based on this observations, and taking into account that an array with a large number of tubes may be assembled in many different configurations, in this work it is proposed a deterministic methodology that allows to created (determine) an optimized assemble subjected to specific operating and building conditions, i. e., for each defined problem on which is specified flow velocity, tube size and materials, fluid, etc., an specific array of tubes will be determined. Following the principals of the Constructal Design theory the velocity and temperature fields were used to define an objective function which once maximized determine the potential tube position.

In other to validate the proposed methodology, the obtained array of tubes, called from this point of Constructal assemble, is compared with the standard aligned and staggered assembles.

\section{NUMERICAL AND MATHEMATICAL FORMULATION}

Geometry and computational domain were created using the open source GMSH software Geuzaine, C. and Remacle, J.-F. (2009). FLUENT software ANSYS (2009) was then used to solve the problem using the FVM.

Construction design has been used to determine the optimized tube assemble. This theory makes possible to describe, in a deterministic form, how system subjected to any king of flow evolve in time. According to this theory, all systems grow (evolve) "building" pathways through regions with less flow resistance. According to Bejan, A. (2000) this behavior occurs both for animate and inanimate systems. Thus, this theory has been successfully applied to different areas such as physics, engineering, biology, social and economical phenomena and sustainability (Bejan, A. (2012).

In this work fluid flow is modeled as bidimensional, laminar and incompressible. All fluid physical properties are considered constants. Continuity, momentum and energy equations, already with the model simplifications are formulated as

$$
\begin{gathered}
\nabla \cdot \vec{v}=0 \\
\rho \frac{D \vec{v}}{D t}=-\nabla p+\mu \nabla \cdot \bar{\tau} \\
\rho c_{p} \frac{D T}{D t}=\nabla \cdot(k \nabla T)+q^{\prime \prime \prime}
\end{gathered}
$$

where $\vec{v}$ is the velocity vector $[\mathrm{m} / \mathrm{s}], \rho$ the density $\left[\mathrm{kg} / \mathrm{m}^{3}\right], \mu$ absolute viscosity $[\mathrm{Pa} \cdot \mathrm{s}], \quad \bar{\tau}$ the stress tensor, $c_{p}$ specific heat at constant pressure $[\mathrm{J} /(\mathrm{kg} \cdot \mathrm{K})]$ and $T$ temperature $[\mathrm{K}]$.

The system formed by Eqs (1)-(3) is solved with FLUENT software. In current solution pressurevelocity coupling is solved with SIMPLE algorithm and second order UPWIND was used for the advective terms discretization. Residues of $10^{-6}, 10^{-6}$ and $10^{-8}$ were used as convergence criterion for mass, momentum and energy equations, respectively. 
Only steady state solution results have been analyzed in this work, however in order to facilitate convergence, a transient numerical solution was employed. In this solution the time step was set to $10^{-3} \mathrm{~s}$ and the steady station condition was obtained when the convective heat transfer coefficient, calculated over the tubes, stops varying with iterations.

Grid independence test has also been calculated with the convective heat transfer coefficient $h$. A grid with just one tube was created and it was considered independent when the error (defined in Eq. (4)) is smaller than $1 \%$.

$$
\text { error }=100\left(\frac{h^{j}-h^{j+1}}{h^{j}}\right)
$$

In Eq. Eq. (4) defines the grid refinement; a larger $j$ indicates a more refined mesh.

The grid independence simulations are summarized in Table 1. Grid M3 was used in all simulations.

Table 1. Grid independence test.

\begin{tabular}{cccc}
\hline Grid & $\mathbf{N}^{\circ}$ of volumes & h & error \\
\hline $\mathrm{M}$ & 9347 & 60.302 & $6.55 \%$ \\
$\mathrm{M}$ & 46821 & 56.351 & $1.55 \%$ \\
$\mathrm{M}$ & 225507 & 55.473 & $0.19 \%$ \\
$\mathrm{M}$ & 495191 & 55.364 & -- \\
\hline
\end{tabular}

The values for $h$ shown in Table 1 were compared with values calculated with empirical correlations suggested by Çengel, Y. A. (2011) and agreed within $5 \%$.

\section{PROBLEM DESCRIPTION}

A dimensionless domain is presented in Fig. 1. It has $69 D$ in $x$ direction and $7 D$ in $y$ direction, being $D$ the tubes diameter. Location where tubes can be positioning is limited by the white region. This geometric constraint guarantees that none of the tubes will be placed in contact with one of the walls and that flow pattern will not be modified by the right wall boundary condition.

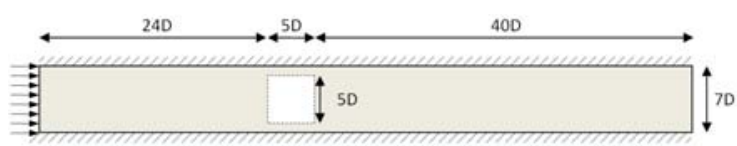

Figure 1. Computational domain.

Four different tube distance constraints have been used in current work. They are:

- free (no constraint)

$-p=1,25 D$

$-p=1,5 D$

$-p=2 D$
The fluid is forced through left wall with prescribed velocity, $U_{\infty}$, and temperature $T_{\infty}$. The temperature at tube surface is $T_{t}$. All walls are considered insulated with no slip flow boundary condition. At the right wall, parabolic boundary condition is specified. Computational domain with all boundary conditions are schematically shown in Fig. 2.

\section{CONTROL FUNCTION AND SOLUTION ALGORITHM}

It is necessary to define a control function that will be used to calculate where in the computational domain each tube should be place. Considering that the heat flux between the tubes and the flow stream may be estimated by

$$
q=h A \Delta T
$$

where $q$ is the heat flux [W], $h$ the convective heat transfer coefficient $\left[\mathrm{W} /\left(\mathrm{m}^{2} \cdot \mathrm{K}\right)\right]$ and $A$ the external superficial tube area $\left[\mathrm{m}^{2}\right]$.

The control function was then formulated based in Eq. (5). Once $h$ is proportional to flow velocity, as larger the velocity larger will be the heat transfer between tubes and fluid. In the same manner, as larger the temperature difference, larger will be the heat transfer. Thus, it is possible to build and dimensionless expression such as

$$
\varphi=\frac{U}{U_{\infty}}\left[\frac{T_{t}-T}{T_{t}-T_{\infty}}\right]
$$

In Eq. (6) it is possible to observe that the temperature term will vary from zero (when $T=T_{t}$ ) to one (when $T=T_{\infty}$ ) and that the velocity term may will assume values larger than one in regions where the velocity is larger than the main stream velocity $U_{\infty}$.

With Eq. (6) it is possible to determine the flow regions where the heat transfer between tube and fluid is potentially more efficient. Consequently it is possible to calculate where a new tube should be positioned.

Array construction will be performed with the following algorithm:

1) define position of the first tube;

2) solve fluid flow and thermal problem (Eqs. (1) to (3));

3) calculate $\varphi$ with Eq. (6);

4) calculate $\varphi_{\max }$ and its coordinates $\left(x_{c}, y_{c}\right)$;

5) create the new geometry with a new tube placed at $\left(x_{c}, y_{c}\right)$;

6) redo steps 2 to 5 until all tubes are positioned. 


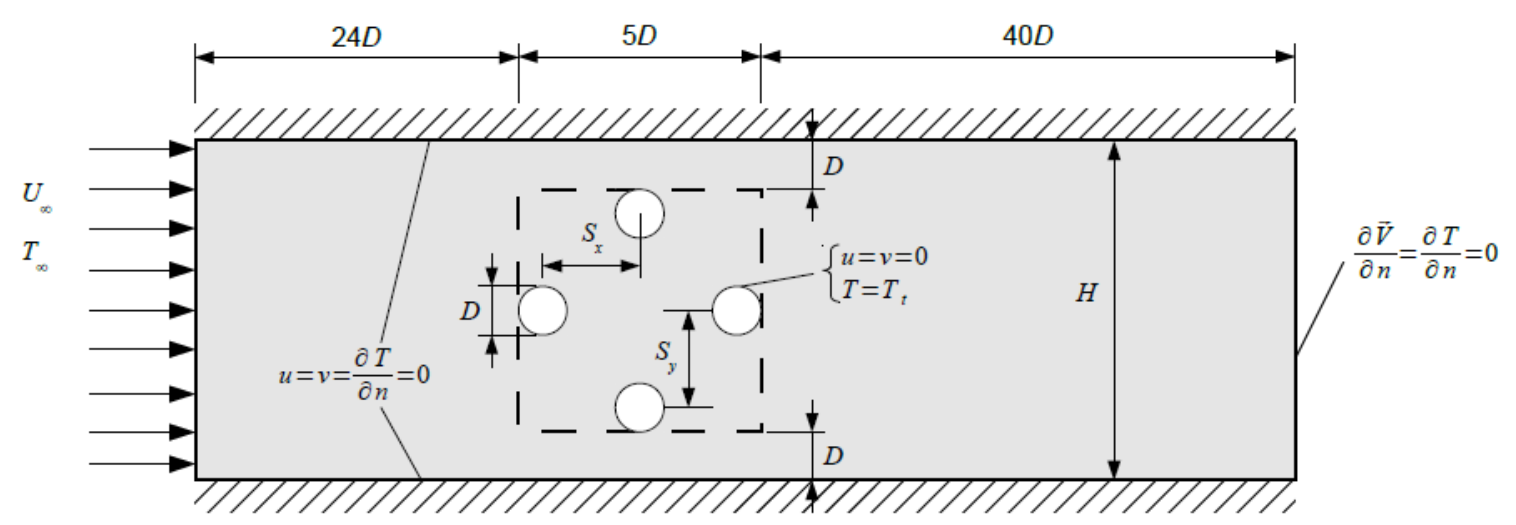

Figure 2. Boundary conditions (not in scale).

\section{RESULTS}

Initially the traditional aligned and staggered assembles (Çengel, Y. A. (2011), Incropera, F. P. et al. (2010)) have been simulated. The objective is to evaluate the heat exchange between tubes and fluid in these classical assembles.

Heat flux entering $\left(q_{\text {in }}\right)$ and leaving $\left(q_{\text {out }}\right)$ the computational domain through left/right walls are evaluated and its difference $(\Delta q)$ is said to be the amount of energy exchanged between tubes and flow stream.

In all simulations the heat flow entering the computational domain is the same and equal to $91.945 \mathrm{~W}$.

Figures 3 and 4 show the scalar field for the control function $\varphi$ for the aligned and staggered assemble, respectively. Regions in red represent higher values of $\varphi$.

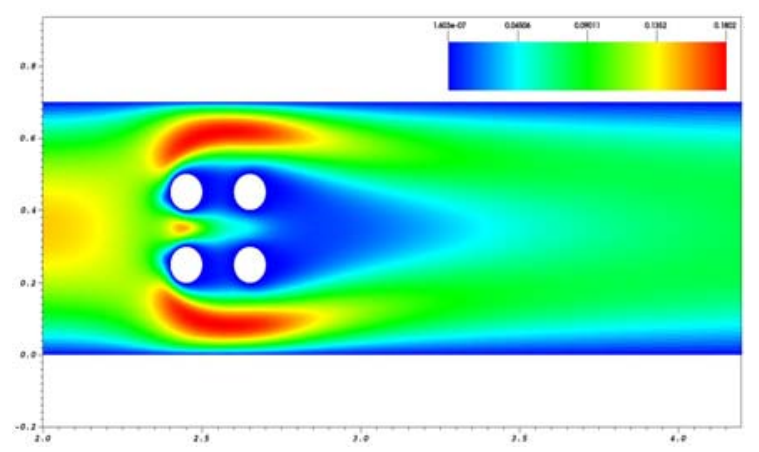

Figure 3. Control function for the aligned assemble.

Heat flux balance $\left(\Delta q=\mathrm{q}_{\text {out }}-q_{\text {in }}\right)$ for aligned and staggered assembles were calculated as $398.98 \mathrm{~W}$ and $540.65 \mathrm{~W}$, respectively.

The absolute value of the control function $\varphi$ is not important, however a qualitative analysis can be done by the observation of Figs. 3 and 4. Regions in read represent the locations where heat exchange is potentially larger while in the blue regions the heat exchange potential is very low. Thus, assembles where many tubes are located in blue regions (compare Fig. 3 and 4) will probably have a lower performance. For example, there two tubes in the blue region of the aligned assemble (Fig. 3) while there is just one tube in the blue region of the staggered assemble (Fig. 4). As shown before, the amount of energy removed from the tubes is $35 \%$ larger in the staggered assemble.

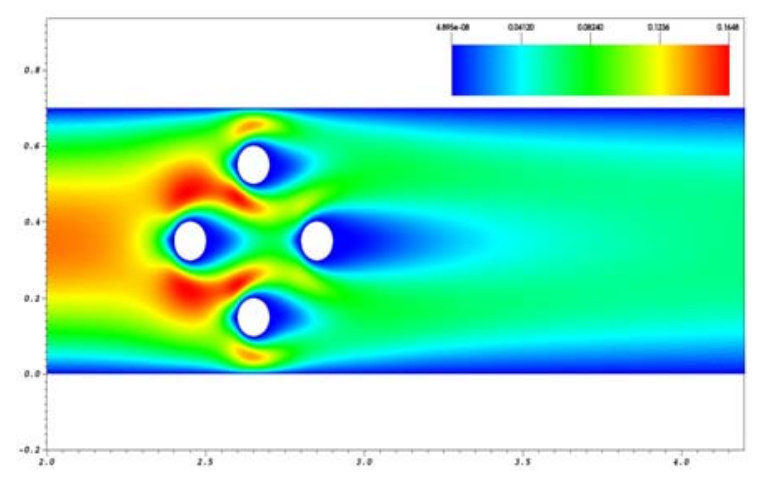

Figure 4. Control function for the staggered assemble.

The Constructal assemble was obtained by initially solving the problem for the geometry with only one tube. This tube was positioned at the middle section between upper and lower walls as show in Fig. 5 (tube 1). With calculated velocity and temperature fields the control function is evaluated and the calculation of its maximum value coordinates will determine the second tube center. After that, flow and thermal problems are again solved and this procedure is repeated till the last tube is positioned inside the computational domain. It is important to highlight that the number of simulations needed to build the Constructal assemble is always equal to the number of tubes.

This procedure was repeated for the four cases: $p=1.25 D, 1.5 D, 2 D$ and free.

In Fig. 5 is shown the Constructal assemble for the case without restriction ( $p$ is free). The numbers inside the tubes in Fig. 5 indicates how (in which order) the tubes were positioned. For this assemble $\Delta q=517.75 \mathrm{~W}$. 


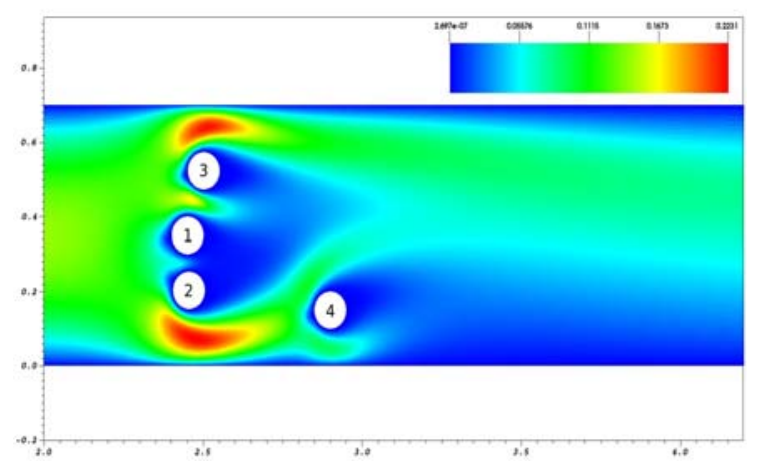

Figure 5. Control function for the free Constructal assemble.

Figure 6 shows the scalar fields of the control function $\varphi$ for the cases with $p=1.25 D$ and $p=1.5 D$. In these cases $\varphi$ calculations results in the same fields and for this reason the tube positions are identical in both assembles. Calculated $\Delta q$ for these two assembles was $522.45 \mathrm{~W}$.

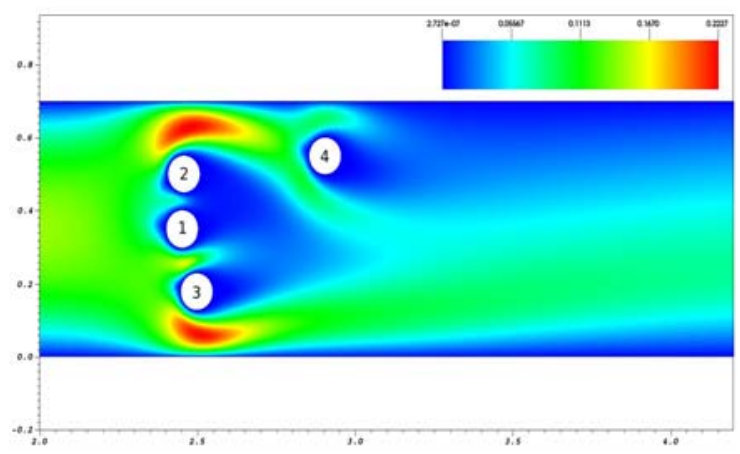

Figure 6. Control function for the free Constructal assemble with $p=1.25 D$ and $1.25 D$.

In Fig. 7 is shown the Constructal assemble for $p=2 D$. In this assemble $\Delta q=548.13 \mathrm{~W}$.

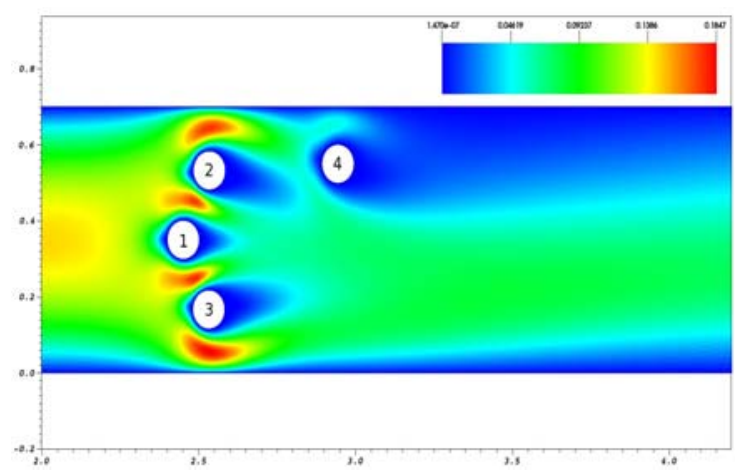

Figure 7. Control function for the free Constructal assemble with $p=2 D$.

In Table 2 results for heat exchange balance is summarized. For this particular solution, the Constructal assemble obtained with $p=2 D$ had the best thermal performance.
Table 2. Four simulated assembles comparison.

\begin{tabular}{c|c|c}
\hline Assemble & $\boldsymbol{q}_{\text {out }}[\mathbf{W}]$ & $\Delta \boldsymbol{q}[\mathbf{W}]$ \\
\hline aligned & 490.927 & 398.982 \\
staggered & 632.410 & 540.645 \\
free & 609.696 & 517.751 \\
$p=1.25 D$ and $p=1.5 D$ & 614.398 & 522.453 \\
$p=2 D$ & 540.078 & 548.133 \\
\hline
\end{tabular}

$q_{\text {in }}=91.945$ in all simulations

\section{CONCLUSIONS}

Thermal performance analysis of the patterns formed with the Constructal Design has shown that setting no constraint to the tubes positioning does not necessarily leads to the most optimized assemble. In this study it was observed that for the specified conditions (flow parameters, fluid and geometry) the best thermal performance was obtained with the assemble in which $p=2 D$. It was also observed that heat exchange is increasing with the increase of $p$, but the free assemble had the worst performance and it is reasonable to suggest that there are an optimal value for $p$, however this possibility was not investigated in this work.

Quantitatively it was calculated that the most efficient assemble $(p=2 D)$ result in a heat exchange between tube and flow stream $5.8 \%$ more efficient than the free assemble and $5 \%$ more efficient than the assembles with $p$ equal to $1.25 D$ and $1.5 D$.

Comparison with the standard assembles, on which distance between tubes was not optimized, the Constructal assemble with $p=2 D$ had shown performances $37 \%$ and $1.5 \%$ more efficient with relation to the aligned and staggered assembles, respectively.

\section{ACKNOWLEDGMENTS}

The authors thanks with gratitude the financial support given by CAPES - Coordenação de Aperfeiçoamento de Pessoal de Nível Superior.

\section{REFERENCES}

ANSYS, 2009, Ansys Fluent 12, Theory Guide.

Bao, Y., Zhou, D., and Huang, C., 2010, Numerical Simulation of flow over Three Circular Cylinders in Equilateral Arrangements at low Reynolds Number by a Second-order Characteristicbased Split Finite Element Method, Computers \& Fluids, Vol. 39, pp. 882-899.

Bejan, A., 2000, Shape and Structure, from Engineering to Nature, Cambridge University.

Bejan, A., 2012, Design in Nature: how the Constructal Law Governs Evolution in Biology, Physics, Technology, and Social Organization, 
Doubleday.

Bello-Ochende, T., Meyer, J. P., and Ogunronbi, O. I., 2011, Constructal Multiscale Cylinders Rotating in Cross-flow, International Journal of Heat and Mass Transfer, Vol. 54, pp. 2568-2577.

Bello-Ochende, T., and Bejan, A., 2005, Constructal Multi-scale Cylinders with Natural Convection, International Journal of Heat and Mass Transfer, Vol. 48, pp. 4300-4306.

Catalano, P., Wangb, M., Iaccarinob, G., and Moinb, P., 2003, Numerical Simulation of the Flow Around a Circular Cylinder at High Reynolds Numbers, International Journal of Heat and Fluid Flow, Vol. 24, pp. 463-469.

Çengel, Y. A., 2011, Heat and Mass Transfer: Fundamentals \& Applications, McGraw-Hill.

Daróczy, L., Janiga, G., and Thévenin, D., 2014, Systematic Analysis of the Heat Exchanger Arrangement Problem using Multi-objective Genetic Optimization, Energy, Vol. 65, pp. 364-373.

Geuzaine, C., and Remacle, J.-F., 2009, Gmsh: A 3-D Finite Element Mesh Generator with Built-in Pre- and Post-processing Facilities, International Journal for Numerical Methods in Engineering, Vol. 79, pp. 1309-1331.

Incropera, F. P., 2010, Fundamentals of Heat and Mass Transfer, John Wiley \& Sons.

Rajani, B. N., Kandasamyb, A., and Majumdara, S., 2009, Numerical Simulation of Laminar Flow Past a Circular Cylinder, Applied Mathematical Modelling, Vol. 33, pp. 1228-1247.

Rocha, L. A. O., Gomes, M. N., Porte, A. P., Galarca, M. M., Acunha Junior, I. C., da Silva, F. M. V., Isoldi, L. A., and dos Santos, E. D., 2013, Constructal Design of Turbulent Forced Convective Flows Over a Pair of Circular Cylinders, in: Proceedings of Constructal Law Conference, Vol. 1, pp. 174-184.

Sarkar, S., and Sarkar, S., 2010, Vortex Dynamics of a Cylinder Wake in Proximity to a Wall, Journal of Fluids and Structures, Vol.26, pp. 19-40.

Stanescu, G., Fowler, A. J., and Bejan, A., 1996, The Optimal Spacing of Cylinders in Free-stream Cross-flow forced Convection, International Journal of Heat and Mass Transfer, Vol. 39, pp. 311-317.

Sumner, D., 2010, Two Circular Cylinders in Cross-flow: a Review, Journal of Fluids and Structures, Vol. 26, pp. 849-899. 